\section{Diagnosis of biliary cystadenoma by peroral video cholangioscopy}
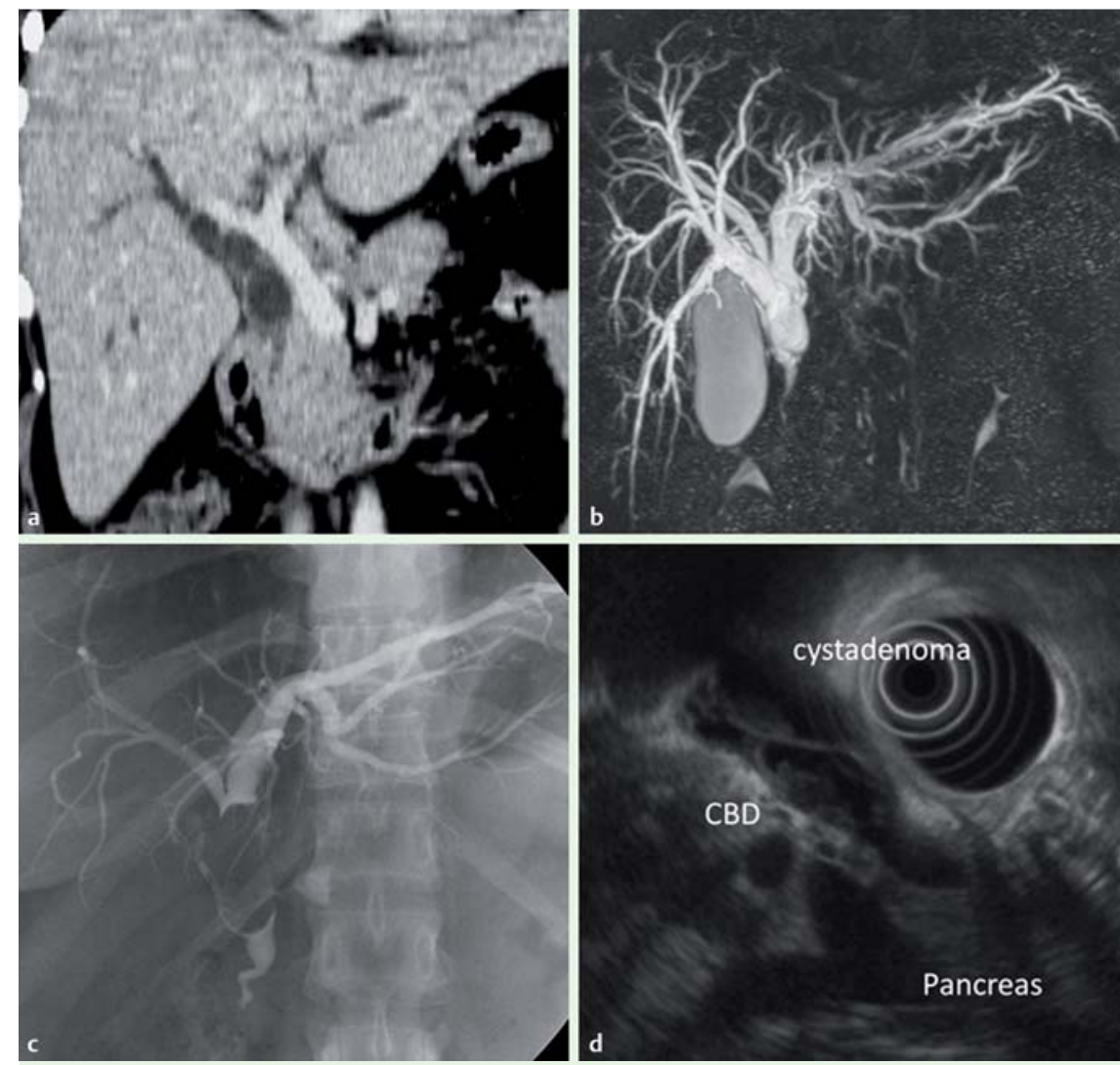

Fig. 1 Longitudinal imaging of the common bile duct (CBD) by various examinations. a Contrast-enhanced computed tomography showed diffuse dilatation of the intrahepatic and common bile ducts. b Magnetic resonance cholangiopancreatography demonstrated irregular dila-

A 31-year-old woman with jaundice was admitted to our hospital. Computed tomography, magnetic resonance cholangiopancreatography, and endoscopic retrograde cholangiography suggested the presence of a solitary cystic lesion in the common bile duct (CBD) ( $\boldsymbol{\bullet}$ Fig. $\mathbf{1} \mathbf{a}, \mathbf{b}, \mathbf{c})$. Subsequent endoscopic ultrasonography (EUS) and intraductal ultrasonography (IDUS) showed a multilocular cystic tumor with cyst-in-cyst pattern in the CBD (๑ Fig. 1 d; $\odot$ Fig.2).

After the patient had recovered from jaundice, peroral video cholangioscopy (POCS) was performed. POCS revealed that the tumor had a flat surface with a fine network of thin vessels, suggesting

tation of the CBD. c Endoscopic retrograde cholangiography demonstrated the elliptic defect in the CBD. $\mathbf{d}$ Endoscopic ultrasonography showed a multilocular cystic tumor in the mid to upper portion of the $C B D$ without signs of infiltrative growth. tors. sidered to originate from the embryonic tissue precursors of the biliary epithelium and to be premalignant lesions.

This is the first report of a diagnosis of extrabiliary cystadenoma by POCS prior to surgery. In this case, POCS showed that the tumor arose from the bile duct wall and had a smooth surface with a fine network of thin vessels, features that are compatible with a benign lesion [5] and are potentially characteristic findings of BCA. POCS clearly depicted the cystic tumor in the CBD. Imaging studies enabled preoperative diagnosis of $B C A$ in the $C B D$. Such tumors are probably better termed ovarian tumor of the bile duct.

Endoscopy_UCTN_Code_CCL_1AZ_2AC

\section{Competing interests: None}

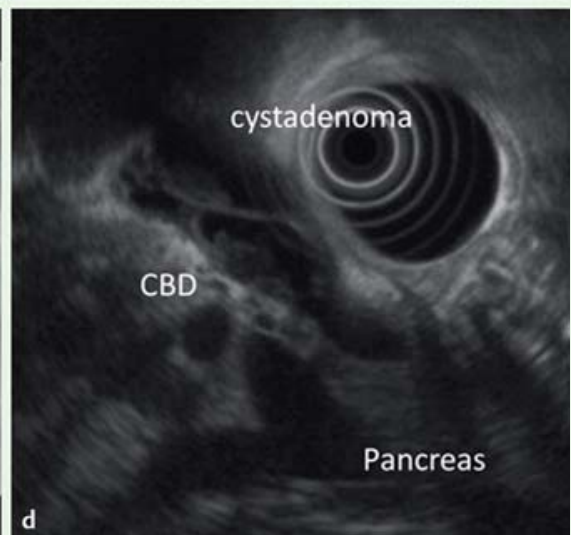

that it was a benign tumor ( Fig.3). Based on these findings, a preoperative diagnosis of biliary cystadenoma (BCA) was made. The patient underwent choledochal resection and subsequent choledochojejunostomy. The resected specimen included a 45-mm multilocular cystic mass in the CBD. Microscopically, the cyst wall was lined by a single layer of high columnar non-neoplastic epithelium, with underlying ovarian-like stroma. Spindle cells in the ovarian-like stroma were positive for progesterone and estrogen recep-

BCAs are rare neoplasms that usually occur in the liver and, less frequently, in the extrahepatic bile ducts [1-4]. They are con-
S. Watanabe ${ }^{1,2}$, T. Sato ${ }^{2}$, K. Hosono ${ }^{2}$, N. Kobayashi ${ }^{2}$, I. Endo ${ }^{3}$, T. Nakayama ${ }^{4}$, Y. Inayama ${ }^{4}$, A. Nakajima ${ }^{2}$, K. Kubota ${ }^{2}$

${ }^{1}$ Division of Gastroenterology, Saiseikai Yokohamashi-Nanbu Hospital, Yokohama, Japan

${ }^{2}$ Division of Gastroenterology, Yokohama City University School of Medicine, Yokohama, Japan ${ }^{3}$ Division of Surgery, Yokohama City University School of Medicine, Yokohama, Japan

${ }^{4}$ Division of Pathology, Yokohama City University School of Medicine, Yokohama, Japan

\section{References}

1 Devaney K, Goodman ZD, Ishak KG. Hepatobiliary cystadenoma and cystadenocarcinoma. A light microscopic and immunohistochemical study of 70 patients. Am J Surg Pathol 1994; 18: 1078-1091

2 Wheelar DA, Edmondson HA. Cystadenoma with mesenchymal stroma (CMS) in the liver and bile ducts. Cancer 1985; 56: 1434-1445

3 Van Steenbergen $W$, Ponette E, Marchal G et al. Cystadenoma of the common bile duct demonstrated by endoscopic retrograde cholangiography: an uncommon cause of extrahepatic obstruction. Am J Gastroenterol 1984; 79: 466-470

4 Umphrey HR, Mel Wilcox C, Vickers SM. Extrahepatic biliary cystadenoma localized to the common bile duct. Surgery 2002; 131 : 587-588

5 Itoi $T$, Osanai $M$, Igarashi $Y$ et al. Diagnostic peroral video cholangiography is an accurate diagnostic tool for patients with bile duct lesions. Clin Gastroenterol Hepatol 2010; 8: 934-938 


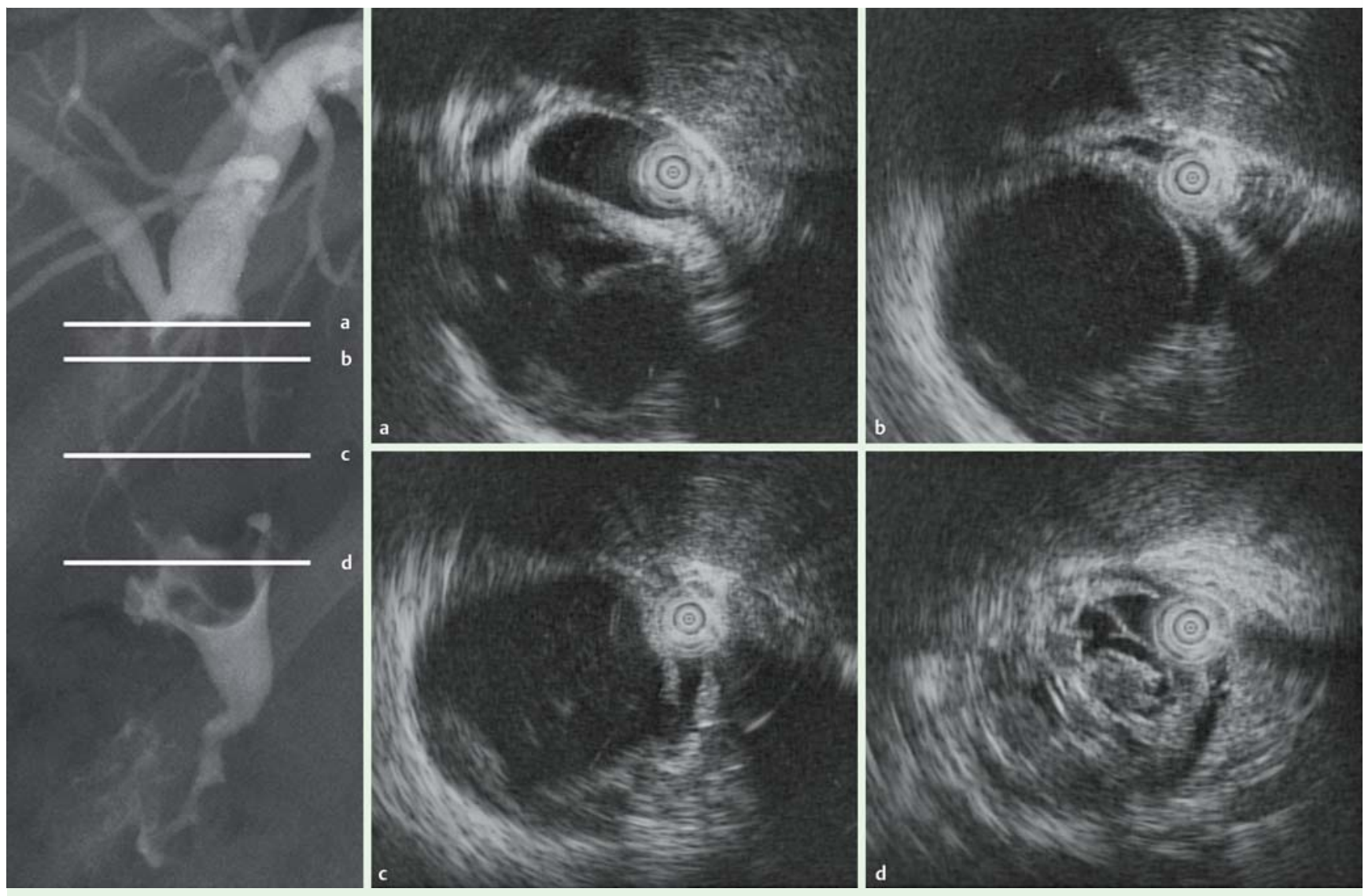

Fig. 2 Intraductal ultrasonography: the multilocular lesion gradually compressed the wall of the common bile duct and finally occluded the lumen.

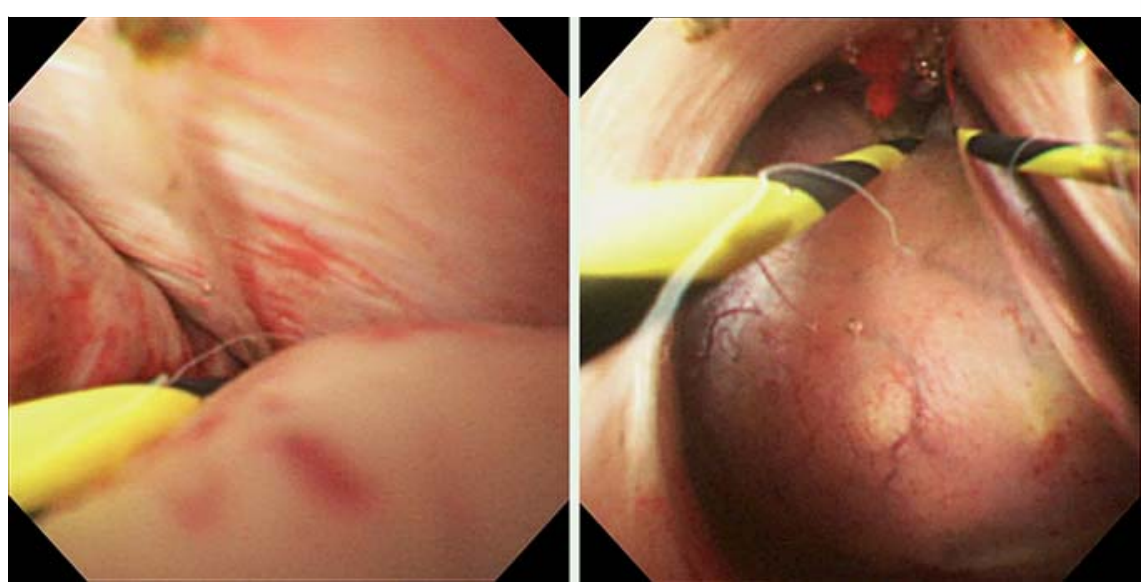

Fig. 3 Peroral cholangioscopy demonstrated a smooth and soft cystic lesion in the common bile duct.
Bibliography

Dol http://dx.doi.org/

10.1055/s-0033-1344416

Endoscopy 2013; 45: E284-E285

(c) Georg Thieme Verlag KG

Stuttgart · New York

ISSN 0013-726X

\section{Corresponding author}

\section{S. Watanabe, MD}

Division of Gastroenterology

Saiseikai Yokohamashi-Nanbu Hospital

3-2-10 Kounandai

Kounan-ku

Yokohama 234-8503

Japan

Fax: +81-45-8328335

swatanabe-ymn@umin.ac.jp 